\title{
Investigation and Implementation Ultra-Low Power PIC-Based Sensor Node Network with Renewable Energy Source and Decision-Making Unit
}

\author{
Batur Alp Akgül1*, Muhammet Fatih Hasoğlu², Bülent Haznedar² \\ ${ }^{1}$ Electronics and Computer Engineering, Institute of Natural Science, Hasan Kalyoncu University, Gaziantep, Turkey \\ ${ }^{2}$ Computer Engineering, Hasan Kalyoncu University, Gaziantep, Turkey \\ Email: *baturalpakgul@gmail.com,mfatih.hasoglu@hku.edu.tr, bulent.haznedar@hku.edu.tr
}

How to cite this paper: Akgül, B.A., Hasoğlu, M.F. and Haznedar, B. (2018) Investigation and Implementation Ultra-Low Power PIC-Based Sensor Node Network with Renewable Energy Source and Decision-Making Unit. Wireless Sensor Network, 10, 41-58.

https://doi.org/10.4236/wsn.2018.102002

Received: January 25, 2018

Accepted: February 25, 2018

Published: February 28, 2018

Copyright $\odot 2018$ by authors and Scientific Research Publishing Inc. This work is licensed under the Creative Commons Attribution International License (CC BY 4.0).

http://creativecommons.org/licenses/by/4.0/

\begin{abstract}
Advancing technology has enabled the production of smaller, more energy efficient and cheaper electronic components. Therefore, previously existing many computer and electronics science-engineering ideas have become feasible. One of them is the technology of wireless sensor networks (WSNs), which has become the realization of the necessary technical requirements applicable today with low energy consumption. First, the sensing tasks and the potential sensor networks applications have explored, and reviews of factors influencing the design of sensor networks have provided. Then, the communication architectures for sensor networks have been outlined. PIC-based microcontrollers have used in the design of the sensor nodes. The design of the sensor node has supported with ultra-low power nanowatt technology for very low-cost design. Processing, memory and wireless communication units have integrated on to the sensor nodes and sensors to be used in the designed system which have allowed to be connected to any kind of sensor node. The designed sensor node's operating system has written with the PIC C language, and PIC operating system has allowed different features such as measuring humidity, temperature, light sensitive and smoke sensor. Computer software has developed for data that can be recorded and monitored from a central location. Decision-making unit has created in the software algorithm and hardware modules for the implementation of decisions taken by the developed sensor nodes. Developed PIC-based sensor nodes have supported a unique voltage unit with renewable energy sources such as solar panel, rechargeable battery, and supercapacitor for energy production and saving. The results of this study are expected to be helpful for the development of WSN especially with re-
\end{abstract}


newable energy sources.

\section{Keywords}

Wireless, Sensor, Energy, Consumption, PIC, Node, Develop, Design

\section{Introduction}

A sensor node can observe conditions of a certain area like temperature, vibration, pressure, sound, humidity, motion, pollutants and so on [1] [2]. Measured values are recorded in a database for further processing purpose. Figure 1 shows a sample wireless sensor network architecture and Figure 2 shows a sensor node structure. In WSNs, researchers focus on wireless sensing nodes which transfer values without being dependent on wires. Moreover, it is easier to replace nodes which have to be observed. WSN's big disadvantages are still limitations in processing power, bandwidth, storage, energy constraints, and supply. The energy of the nodes is the primary measure that dominates WSNs due to its profound impact on its features such as network operational lifetime, connectivity and routing protocols. So renewable energy sources are very crucial to extend life spans such as solar power and rechargeable battery. Therefore, the main goal of this study is to find the most suitable hardware and software for the sensor node design for low power consumption utilizing renewable energy sources with a long network lifespan. Another important goal of this study is to develop software though which data can be recorded and monitored from a central location. Last but not least important goal is to develop a decision-making in the software algorithm and hardware unit for the implementation of decisions made by the developed sensor nodes.

In this study, an alternative solar panel power has used with rechargeable battery and supercapacitor with unique power regulator. Ultra-low powered sensor

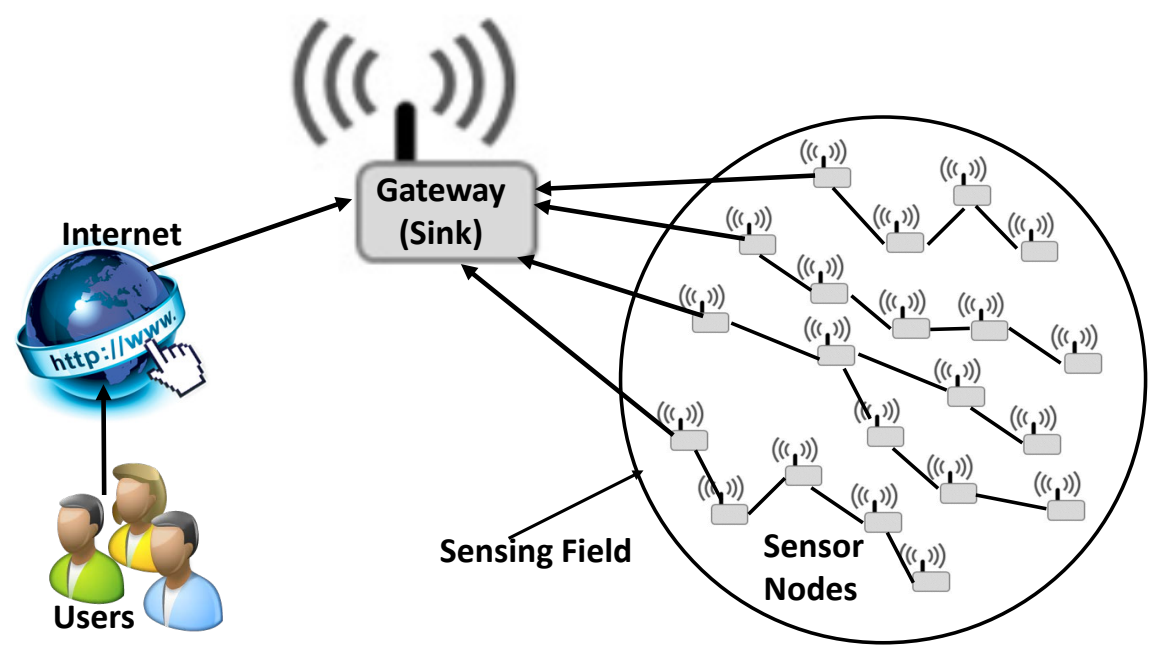

Figure 1. Wireless sensor network architecture. 


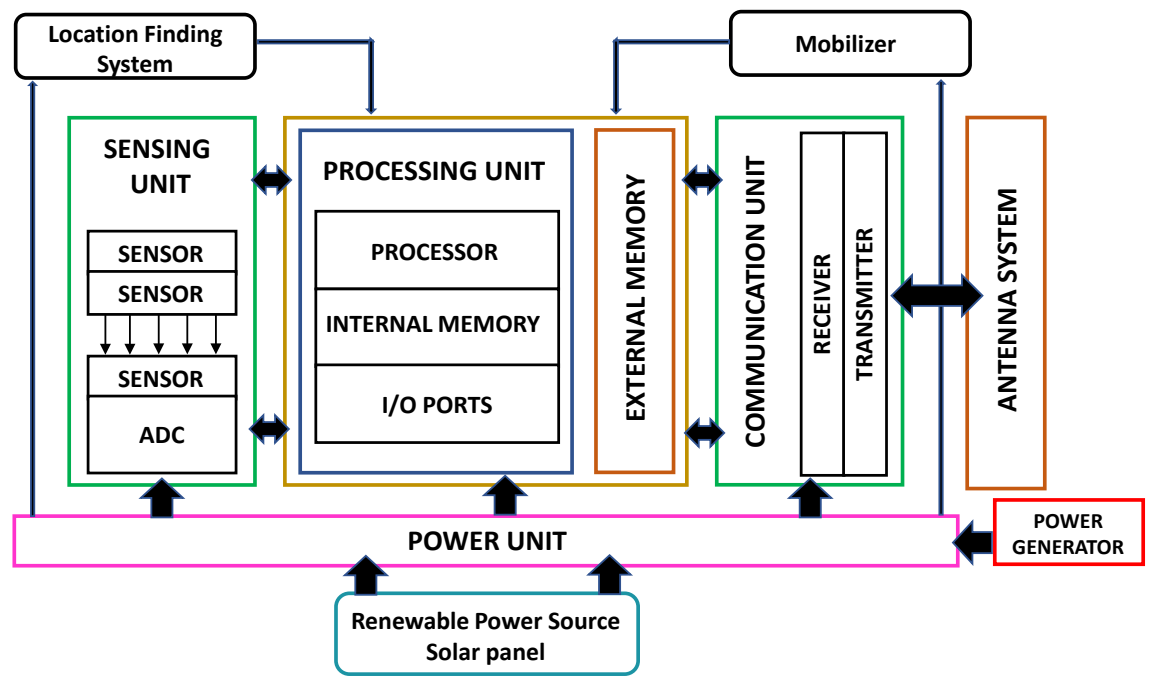

Figure 2. Wireless Sensor Network structure.

node network was previously designed. Details of our studies compared to the previous studies are given in the coming sections and the paper is organized as follows. Section 2 presents literature research, Section 3 presents hardware components of the designed sensor node. Section 4 describes the software components of the designed sensor node. Section 5 presents results and discussions. Section 6 concludes the paper.

\section{Literature Review}

Previous studies on WSNs available in literature presented in this section. Thiemo Voigt, Hartmut Ritter, Jochen Schiller [3], utilized solar power in wireless sensor networks. They have presented two protocols for solar aware routing. Solar-aware routing preferably routes traffic via nodes powered by solar energy. Their simulations of two solar-aware protocols show that they could provide significant energy savings in many scenarios. Results suggest that utilizing solar power in wireless sensor networks is efficient and feasible. The design has named "Utilizing Solar Power in WSNs". Matthew Martino and Jordan Varley [4] have developed a Wireless Sensor Node Powered by a PV/Supercapacitor/ Rechargeable Battery Trio. Their device consists of the WSN with BQ25504-Ultra Low Power Boost Converter with Battery Management for Energy Harvester Application. The design has named "A Wireless Sensor Network Powered by a PV/Supercapacitor/Battery Trio". Tuncay Soylu from Trakya University [5], has done researches about wireless sensor network applications and designed a sensor node that was used for temperature measurements in the medical field. $\mathrm{He}$ called his study "Wireless Sensor Networks Applications and Design of Sensor Node". Omer Ozcan has conducted researches on wireless sensor network applications and he has designed a sensor node [6]. The designed system allows the various software applications and different types of sensors. Internal light on the system LDR sensor and temperature LM35 sensor, 9-pin system has been inte- 
grated into an expansion slot. Sensor nodes have the very simple circuit structure. Mahmoud Shaker and Alaa Imran [7] have developed a WSN based microclimate monitoring system for greenhouse plants with a new irrigation system. System deployment includes multi WSN each one was responsible to monitor and control one row of plants inside the greenhouse. The system mainly consisted of two node types; sensing node and control node.

\section{PIC-Based Sensor Node Platform}

Our designed system consists of sensor node hardware, transmitter (master) sensor nodes, and one receiver (slave) sensor node. The proposed system is composed of a PIC microcontroller, power regulator, wireless transmitter and receiver modules, LED indicators and relay units for Decision-Making. Sensors are integrated on the master sensor nodes (temperature, humidity, gas-smoke). Figure 3 shows image of the designed WSN platform and Figure 4 shows image of the designed sensor node's hardware. Designed wireless sensor nodes have been tested in a real environment with a normal battery, rechargeable battery and solar panel. The designed wireless sensor node's main features are presented in Table 1 and details are given in section 3.

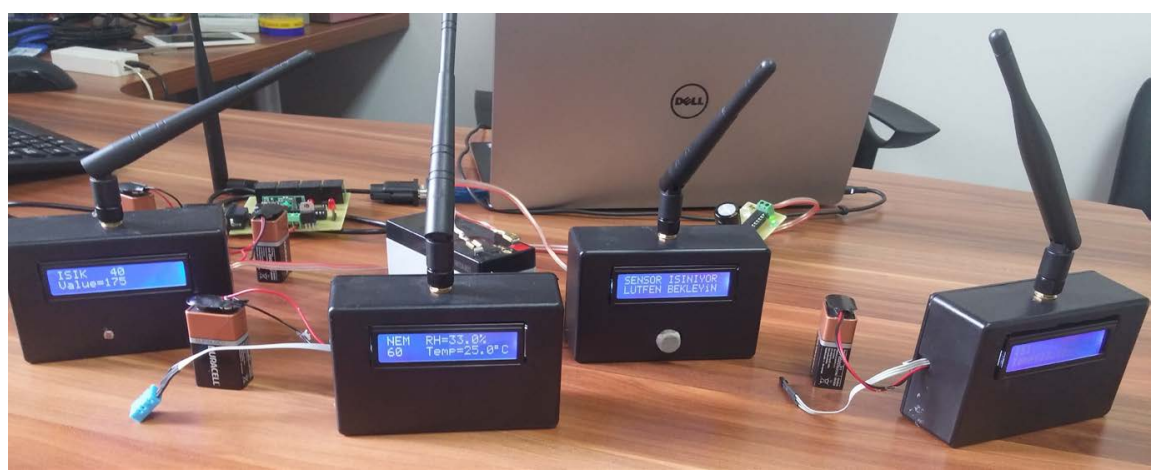

Figure 3. Overview of the designed WSN platform.

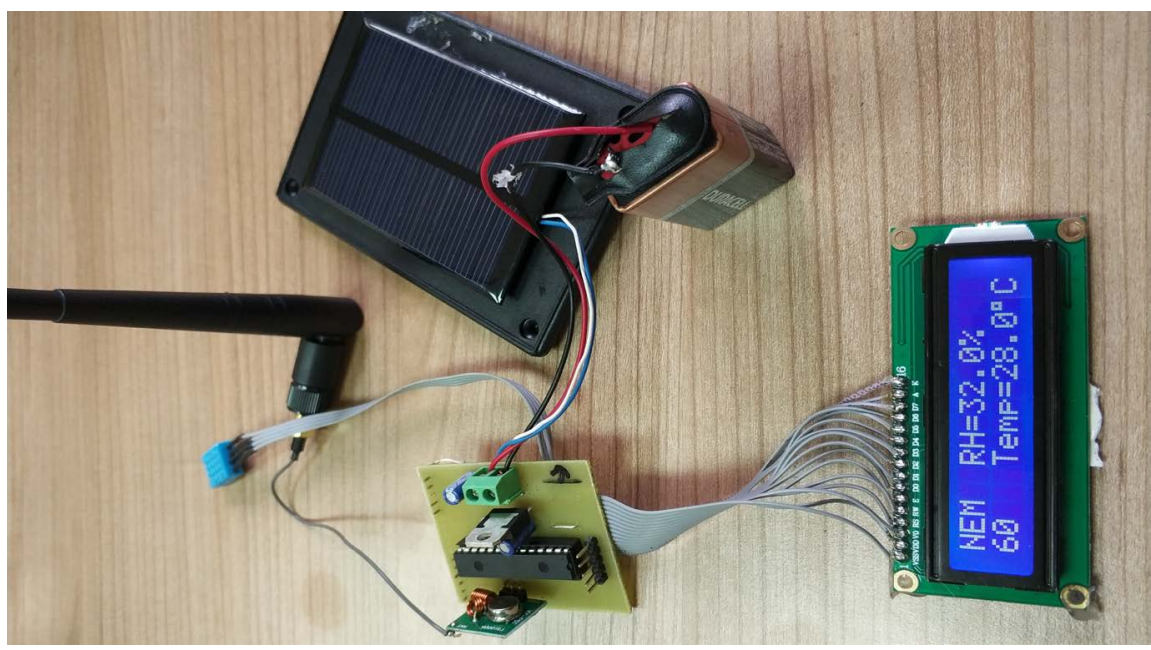

Figure 4. Overview of the designed sensor node's hardware. 
Table 1. Designed sensor node types and used components.

\begin{tabular}{ccccccc}
\hline \multicolumn{7}{c}{ DESIGNED WIRELESS SENSOR NODES } \\
\hline Designed & Used & Used & Voltage & Used & Rechargeable & Solar \\
Sensor Node Type & Sensor & PIC & Regulator & LCD Panel & Battery \& SC & Panel \\
\hline Receiver Node & $\mathrm{x}$ & PIC16F886 & LM7805 & X & $\square$ & $\square$ \\
Transmitter Node & DHT11 & PIC16F886 & LM7805 & 1602A LCD & $\square$ & $\square$ \\
Transmitter Node & LM35 & PIC16F886 & LM7805 & 1602A LCD & $\square$ & $\square$ \\
Transmitter Node & MQ2 & PIC16F886 & LM7805 & 1602A LCD & $\square$ & $\square$ \\
Transmitter Node & LDR & PIC16F886 & LM7805 & 1602A LCD & $\square$ & $\square$ \\
\hline
\end{tabular}

\subsection{Hardware Components}

WSN platform uses a PIC microcontroller of model number PIC16F886. This microcontroller has 28 Pin Flash-Based, 8-Bit CMOS Microcontroller with NanoWatt Technology. The PIC has an external oscillator, 4K EEPROM, ADC, timers, PWM pulse-width modulation, 1 EUSART. In order to keep power consumption laws, no external memory unit was used [8]. FS1000A module was used as wireless transmitter unit, and XY-MK module was used as a wireless receiver. FS1000A and XY-MK modules work in $433 \mathrm{MHz}$ band and can run on 10 different channels. In order to read data on the sensor node without a computer program, 1602A LCD panel was integrated [9]. In Table 2 the comparison data provided with the designed sensor node and the other sensor nodes are shown. In order to transfer data to the computer database, RS232 connection port and RS232 transceiver (MAX232) were integrated. In order to increase wireless communication signal, antennas were integrated to the sensor node system in both receiver and transmitter. JQC-3FC/T73 model relays are designed to sensor nod receiver system for electrically activated switch purposes. DHT11 and LDR temperature and humidity sensor, MQ-2 gas sensor, and LM35 precision centigrade temperature sensor are used on the master sensor nodes [12] [13] [14]. Images of designed master and slave sensor nodes are shown in Figure 5 and Figure 6, respectively. Detailed circuit drawing of master and slave nodes are shown in Figure 7 and Figure 8, respectively.

\subsection{Power Management}

Power unit provides power to the sensor node is shown in Figure 9. The LM317 voltage regulator was implemented on the power unit which includes on-card regulation for elimination of noise and distribution problems. Regulators can deliver up to 1.5A of output current and Output Voltage Range Adjustable from $1.25 \mathrm{~V}$ to $37 \mathrm{~V}$ [15]. PIC16F886 microcontroller can operate within the range of $2.0 \mathrm{~V}-5.5 \mathrm{~V}$. FS1000A and XY-MK modules can also operate within the range $3.5 \mathrm{~V}-12 \mathrm{~V}$. Therefore, the system works on $3.5 \mathrm{~V}$ because PIC microcontroller and wireless communication modules support this voltage. Low-power routing algorithms and low power media access (MAC) protocols can decrease energy consumption and increase battery life. 


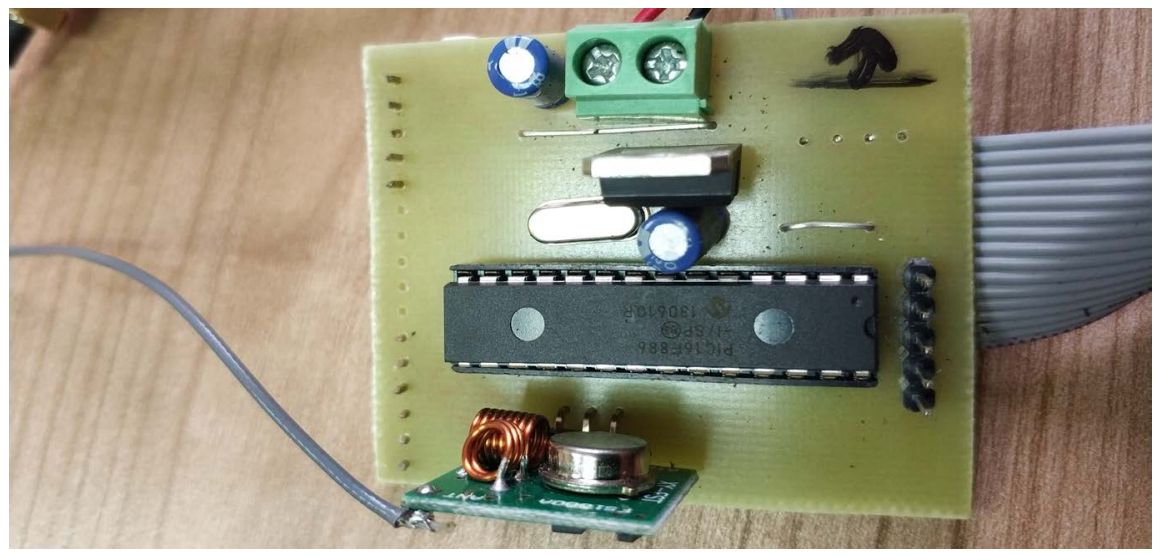

Figure 5. PIC-based master (transmitter) sensor node.

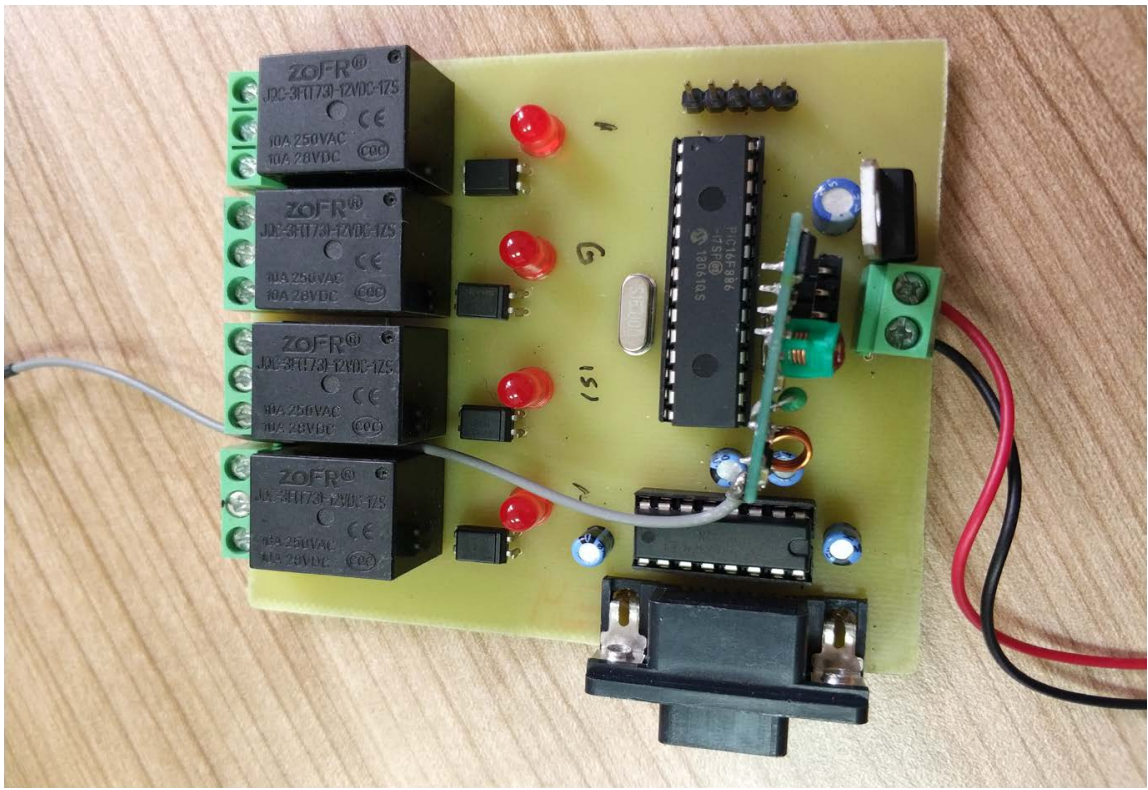

Figure 6. PIC-based slave (receiver) sensor node.

Table 2. Feature comparison of sensor nodes.

\begin{tabular}{ccccccc}
\hline \multicolumn{7}{c}{ SENSOR NODES FEATURES } \\
\hline Mote Platform & Controller & Bus & Clock & RAM & Flash & EPROM \\
\hline TelosB/Tmote Sky [10] & TI MSP430F1611 & 16-bit & $4-8 \mathrm{MHz}$ & $10 \mathrm{~K}$ & $48 \mathrm{~K}$ & $1 \mathrm{M}$ \\
IMOTE [11] & ARM7 & 32-bit & $12 \mathrm{MHz}$ & $11 \mathrm{~K}$ & $512 \mathrm{~K}$ & None \\
MicaZ/Mica2 [10] & Atmel Atmega 128L & 8-bit & $8 \mathrm{MHz}$ & $4 \mathrm{~K}$ & $128 \mathrm{~K}$ & $512 \mathrm{~K}$ \\
Tmote Sky [11] & MSP430F & 16-bit & $8 \mathrm{MHz}$ & $10 \mathrm{~K}$ & $48 \mathrm{~K}$ & $1 \mathrm{M}$ \\
SHIMMER [10] & TI MSP430F1611 & 16-bit & $4-8 \mathrm{MHz}$ & $10 \mathrm{~K}$ & $48 \mathrm{~K}$ & None \\
EZ-RF2480/2500 [11] & TI MSP430F2274 & 16-bit & $16 \mathrm{MHz}$ & $1 \mathrm{~K}$ & $32 \mathrm{~K}$ & None \\
IRIS Atmel [10] & Atmega1281 & 8-bit & $8 \mathrm{MHz}$ & $8 \mathrm{~K}$ & $640 \mathrm{~K}$ & $4 \mathrm{~K}$ \\
Mica2Dot [11] & Atmega1281 & 8-bit & $4 \mathrm{MHz}$ & $4 \mathrm{~K}$ & $128 \mathrm{~K}$ & $512 \mathrm{~K}$ \\
Sun SPOT [10] & Atmel AT91RM9200 & 32-bit & $180 \mathrm{MHz}$ & $512 \mathrm{~K}$ & $4 \mathrm{M}$ & None \\
Our Platform & PIC16F886 & 8-bit & $20 \mathrm{MHZ}$ & $368 \mathrm{~K}$ & $14 \mathrm{~K}$ & $256 \mathrm{~K}$ \\
\hline
\end{tabular}




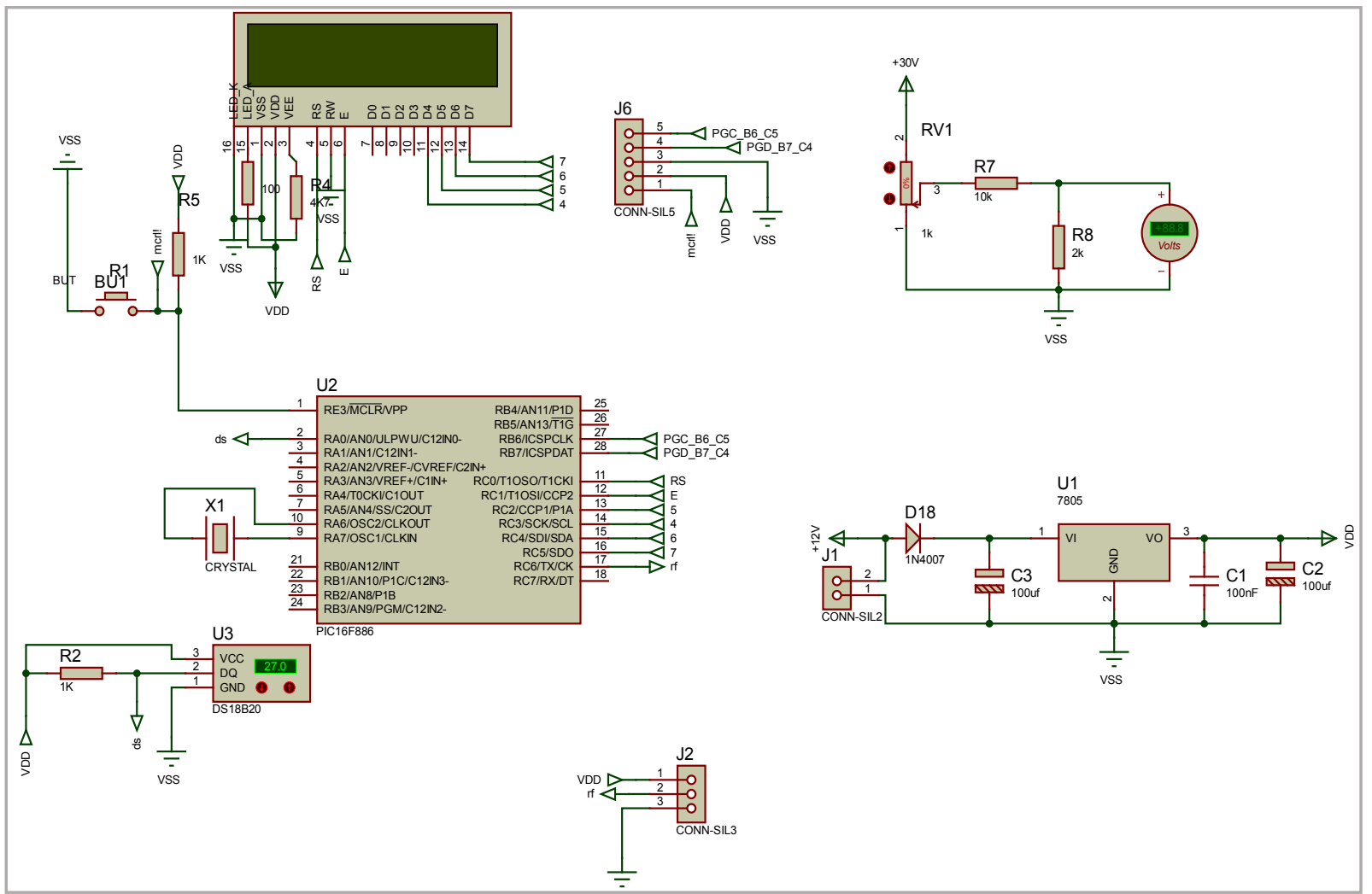

Figure 7. Schematic diagram of the transmitter (master) node.

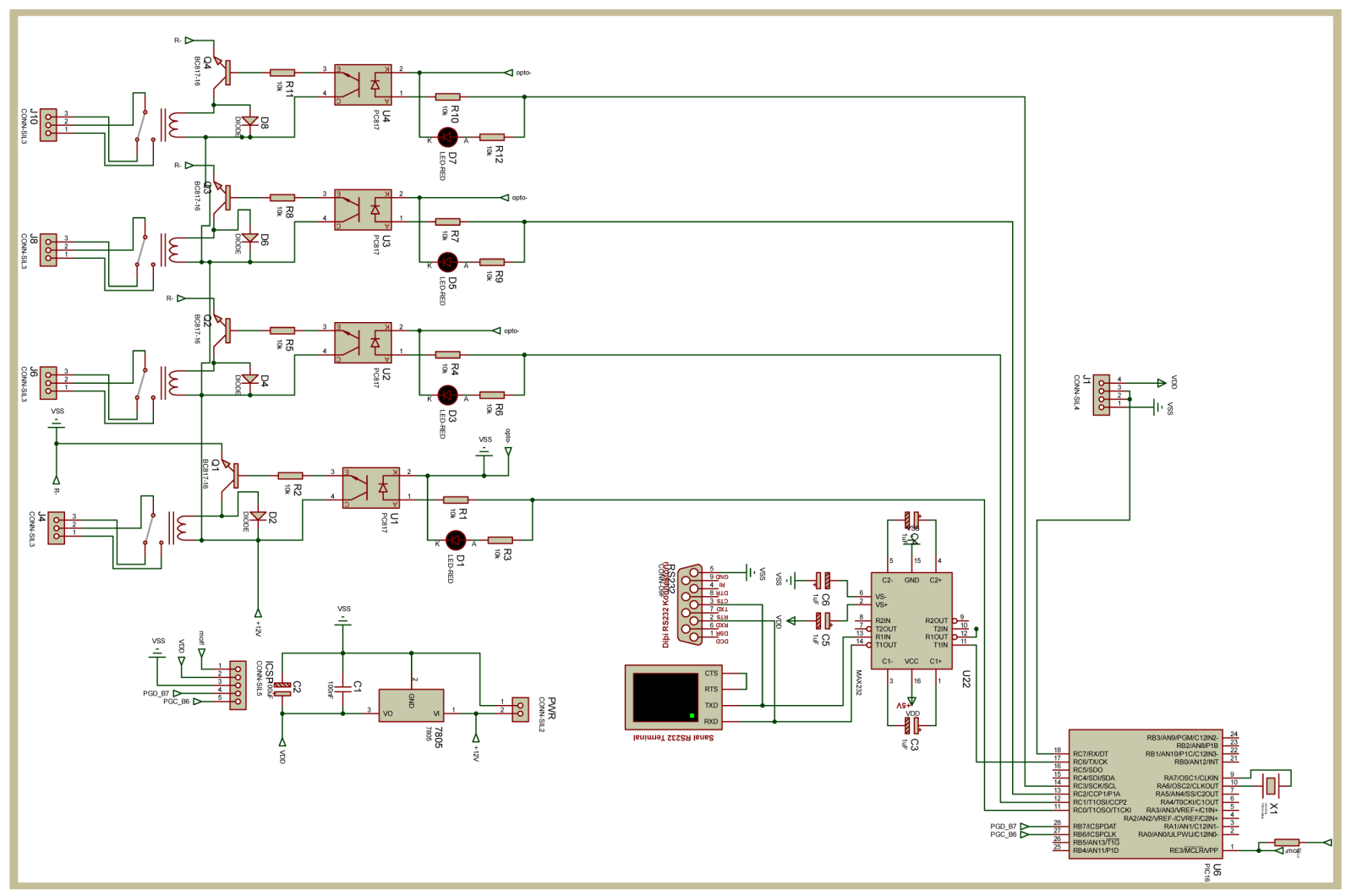

Figure 8. Schematic diagram of the receiver (slave) node. 


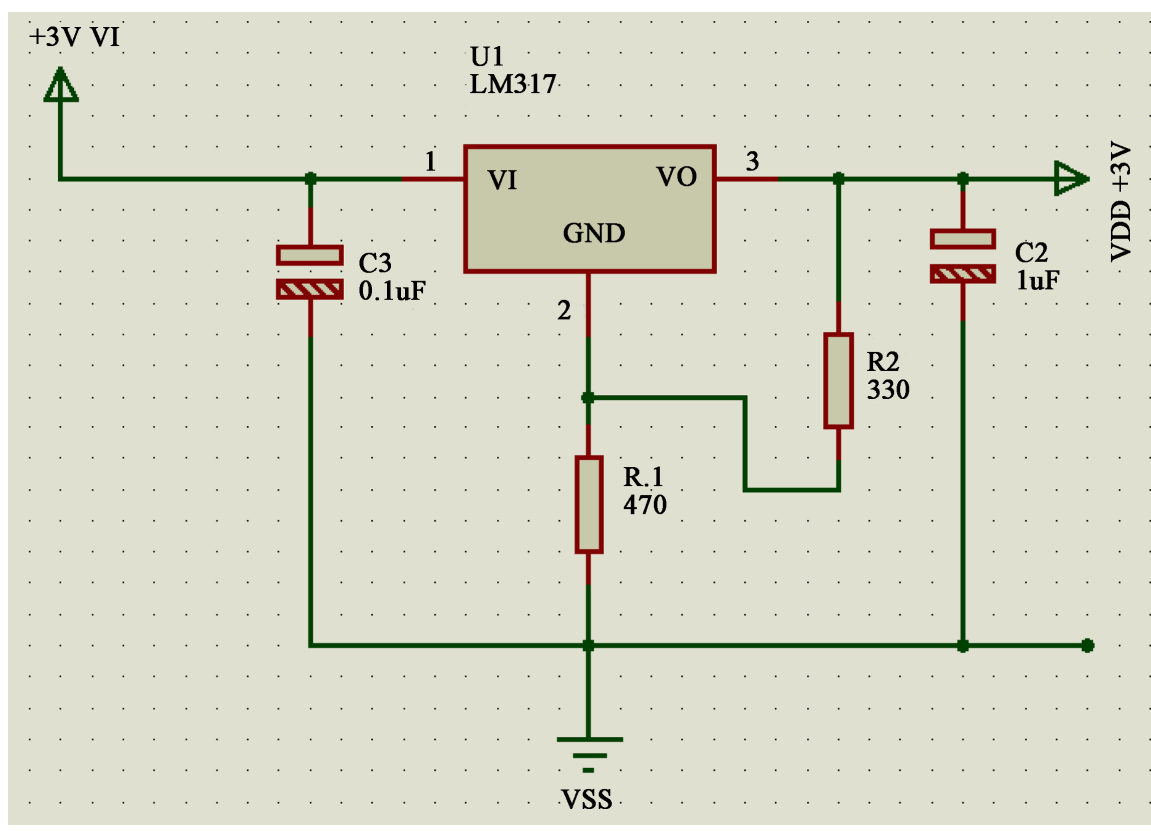

Figure 9. Power Regulator circuit drawing of the designed WSN platform.

\subsection{Energy Consumption}

In WSN, receiving and transmitting power consumptions can vary depending on the sensor node design technics and components. As seen in Table 3, RF433 provides the least energy cost in wireless communication technologies. Therefore, RF433 technology is preferable in the design of sensor nodes.

\subsection{Renewable Energy Management}

Designed DC converter provides an interface to connect to a solar panel, a rechargeable battery, and a supercapacitor that provides efficiency across all operating conditions. Designed DC Converter uses less than $470 \mu \mathrm{A}$ of current, and power unit has ability to harvest available solar energy by using the solar array of panels to support all required components. The obtained energy is stored in a $5 \mathrm{~F}$ supercapacitor and a $350 \mathrm{mAh}$ lithium-ion battery. The rechargeable battery is used as an energy storage device for all sensor nodes. WSN features an ability to receive solar energy from the outdoor environment and deliver this energy to supercapacitor and battery storage devices. Figure 10 contains the block diagram of the energy storage and delivery module, also shows that combination of rechargeable batteries and solar panels. $350 \mathrm{mAh}$ rechargeable battery and one classic bidirectional converter method are used. Designed panel power unit coordinates power storage and delivery between a small solar array, and rechargeable batteries with a supercapacitor, ensuring long network life.

\subsection{Medium Access Control Protocols and Routing Protocols}

Medium Access Control (MAC) protocol determines interaction with devices and MAC protocols are to avoid collisions so that two interfering nodes do not 


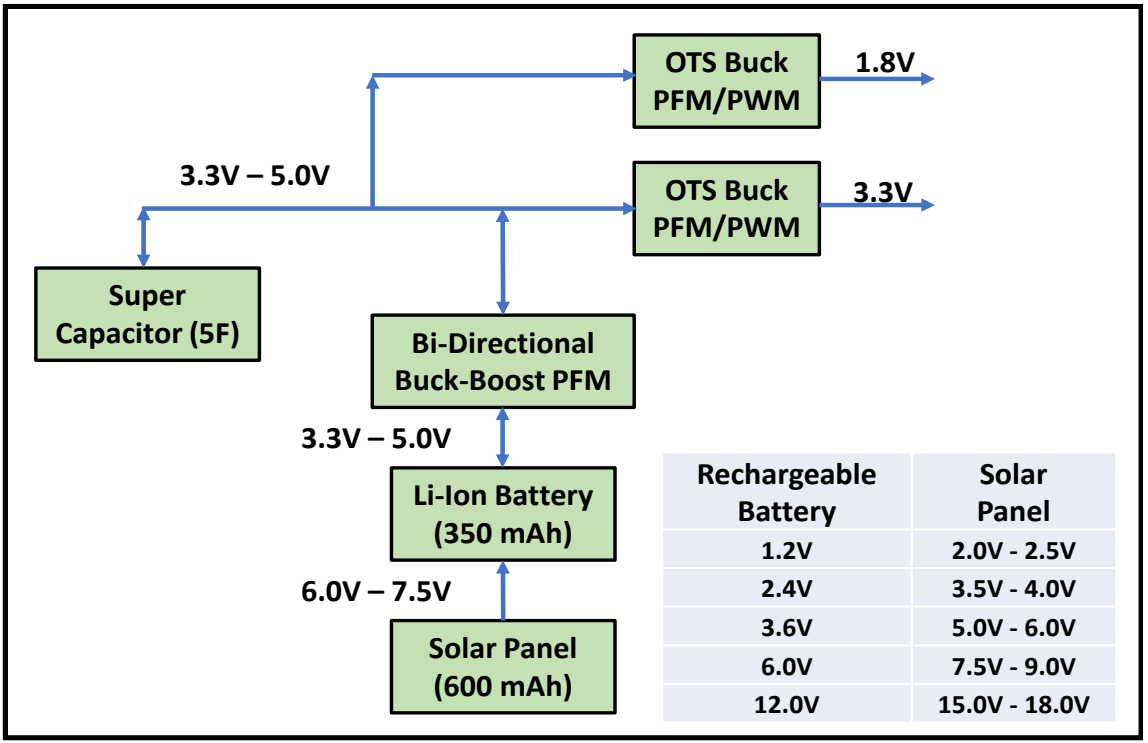

Figure 10. Energy module and Solar panel DC reference table.

Table 3. Power consumption of wireless interfaces [16].

\begin{tabular}{cccccc}
\hline \multirow{2}{*}{ RF Module } & \multicolumn{5}{c}{ POWER CONSUMPTION $(\mathrm{mW})$} \\
\cline { 2 - 6 } & Sleep & Idle & Wake-up & $\mathrm{Tx}$ & $\mathrm{Rx}$ \\
\hline RF433 & $\mathrm{X}$ & 30.14 & 12 & 75.57 & 31.7 \\
ZigBee & 0.165 & 40.56 & 22 & 163.74 & 89.66 \\
Bluetooth & $\mathrm{X}$ & 18.08 & 96.23 & 199.05 & 185.32 \\
Wi-Fi & $\mathrm{X}$ & 61.02 & 66.21 & 67.44 & 63.91 \\
\hline
\end{tabular}

transmit at the same time. MAC protocol types and variants can be adapted to WSNs. Used mechanisms in the MAC protocols are suitable for WSNs. Another important point is that the MAC protocols provide energy efficiently to the WSNs. We consider energy consumption as an important constraint; MAC protocols can be contributed to the reduction of energy consumption. In Timeout MAC (T-MAC) protocol, there is no regular sleep-wake cycle. Sleeping operation takes place when sensor node is unable to obtain data in a certain time period. Waking can start with the start of sending data or when the sleeping time expires. Sensor nodes have a certain time limit for sleeping. In Traffic Adaptive Medium Access (TRAMA) protocol, sensors become synchronized by waiting for each other. When the media is empty, the sensors have access to the environment. In this study, a hybrid MAC protocol was used, this protocol is combined with T-MAC and TRAMA protocols. The hybrid protocol was obtained by making changes on the T-MAC protocol. When the sensor node wakes up, it does not start to send data, instead, it just waits until discharging of the medium. Thus, a sensor node can adapt itself to the traffic and it could also be possible to sleep some time then wake another time. Figure 11 shows the working system of the hybrid protocol. 


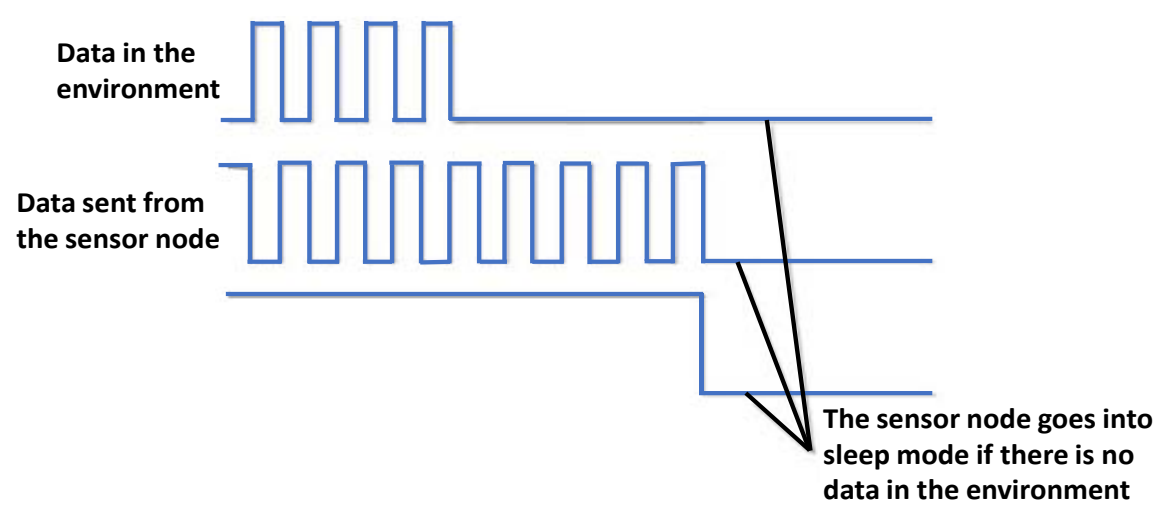

Figure 11. TRAMA MAC hybrid working system.

A routing algorithm determines the way of data transfer. Routing algorithms in WSNs are controlled by software. Point to point direct diffusion routing scheme is used as routing protocol which means designed WSN uses direct communication to slave (receiver) nodes and slave node sends data to the base station. According to this algorithm, data coming out of a sensor node will reach directly receiver sensor node. Each transmitter sensor nodes can send its own data to receiver sensor node in many times. Figure 12 pictorially demonstrates this process. Direct transmission involves energy consumption for sensing and transmission only. Energy is not consumed for receiving as direct transmission does not involve receiving data from any other nodes.

\section{Software Development}

In WSN, software issues can be categorized into two headings. One of this is the computer software that will monitor the PIC sensor nodes and collect and record the data from sensor nodes, the other small embedded PIC operating system (software) that control and manages sensor nodes. All operations of sensor nodes are managed by the operating system and operating system which has been developed with PIC-C language. The developed PIC16F886 sensor node operating system was responsible to determine routing algorithm and MAC protocol, sending data retrieval, data processing, reading data from analog and digital sensors and memory management, communications between sensor nodes etc. For wireless communication, microcontroller software uses packet structure and the data stream is in bytes. If the network gets busy, data should wait in microcontroller's memory for a while. In the system, data from the sensor and all the data from the other sensors are collected in a single queue. Each piece of data from the sensors is transferred to the queue as a byte. The data reading time of the sensors is made using timers in the software. The data is transferred to the queue by reading the sensors at specific time intervals. Data flows are controlled by cutting. The incoming data is transmitted to the queue for transmission via the cutting process. Diagram of data delivery system to other sensors or base station is shown in Figure 13. WSNs do not have any monitoring screen unit on the sensor nodes in many applications due to high cost and 


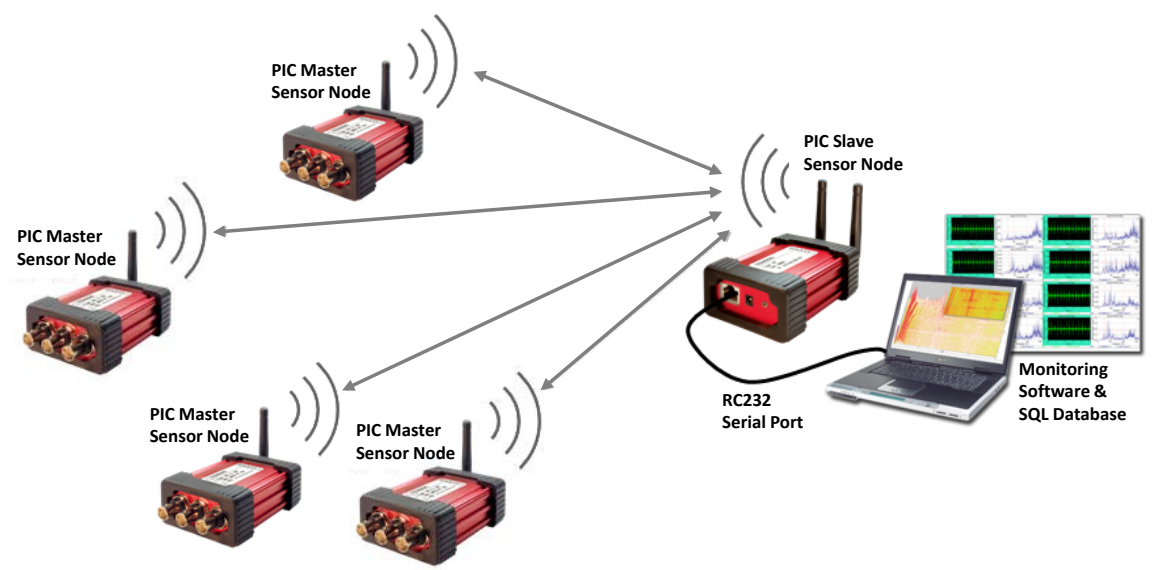

Figure 12. Point to point routing algorithm, direct communication.
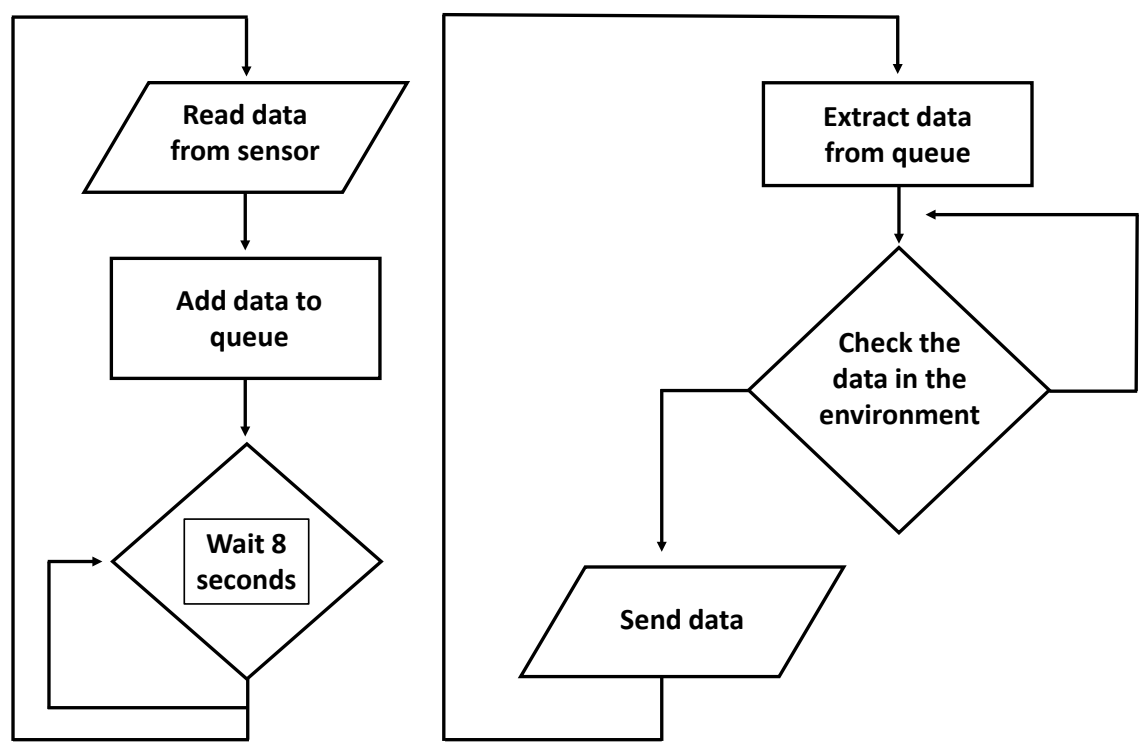

Figure 13. Data transfer and data delivery diagrams of software.

energy saving (in our designed sensor nodes, we have integrated LCD panel to check the results). But all the applications, the data needs to be displayed and recorded in the database, and obtained data could be observed and processed. In this study, an application on the Microsoft Visual Studio and C \# language is developed to monitor and save the data in the database. The interface of the developed application and monitoring process is shown in Figure 14 and Figure 15 shows the SQL Databases with tables and stored data.

\section{Results and Discussions}

Four transmitters (master) nodes designed to monitor and examine the conditions of environment, one receiver sensor node (slave) designed to collect data and send to computer program via the RS232 port with relay unit. The communication module is an RF433 wireless transmitter unit with an antenna. Data and control unit includes a PIC microcontroller 16F886. Designed wireless sensor 


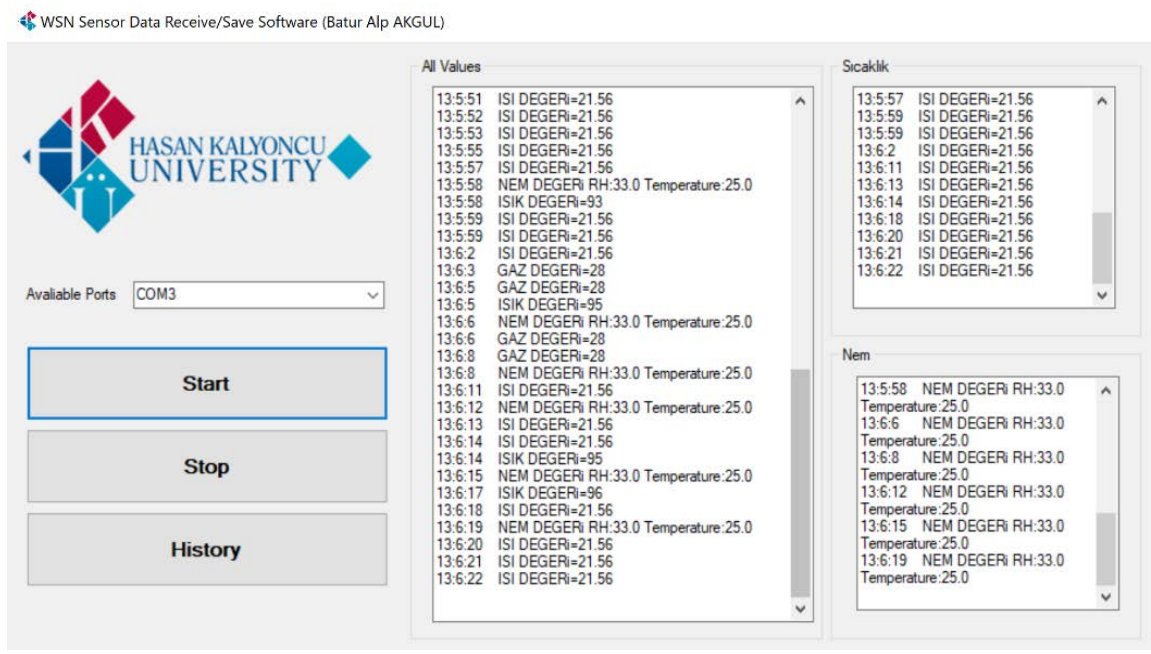

Figure 14. Interface of developed application and monitoring process.

\begin{tabular}{|c|c|c|c|}
\hline Object Explorer & DESKTOP-U & NDB - dbo.ISIK $\rightleftharpoons$ & \\
\hline 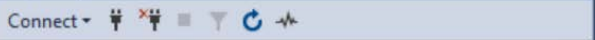 & ID & Tarih & ISIK \\
\hline$\square$ DESKTOP-UA336EPIMSSQLSERVER1 (SQL Server 14,0.1000.16 — & - & 2018-01-09 12:4... & 50,00 \\
\hline$\boxminus \boxminus$ Databases & 2 & 2018-01-09 12:4... & 50,00 \\
\hline$\boxplus$ System Databases & 3 & 2018-01-09 12:4 ... & 51,00 \\
\hline $\begin{array}{l} \pm \text { Database Snapshots } \\
\text { WSNDB }\end{array}$ & 4 & $2018-01-0912: 4 \ldots$ & 52,00 \\
\hline $\begin{array}{l}\boxminus \text { WSNDB } \\
\boxplus \text { Database Diagrams }\end{array}$ & 5 & 2018-01-09 12:4... & 51,00 \\
\hline$\boxminus$ Tables & 6 & 2018-01-09 12:4... & 51,00 \\
\hline It System Tables & 7 & 2018-01-09 12:4... & 51,00 \\
\hline (1) FileTables & 8 & 2018-01-09 12:4... & 55,00 \\
\hline $\begin{array}{l}\boxplus \pm \text { External Tables } \\
\oplus \pm \text { Graph Tables }\end{array}$ & 9 & 2018-01-09 12:4... & 56,00 \\
\hline 甲囲 dbo.GAZ & 10 & 2018-01-09 12:4... & 57,00 \\
\hline (1) dbo.|S| & 11 & 2018-01-09 12:4... & 58,00 \\
\hline 甲囲 dbo.ISIK & 12 & 2018-01-09 12:4... & 56,00 \\
\hline$\boxplus$ 团 dbo.Nem & 13 & 2018-01-09 12:4... & 43.00 \\
\hline
\end{tabular}

Figure 15. SQL database with tables and stored data.

nodes have been tested in a real environment with a normal battery and a rechargeable battery. The data obtained from the environment were observed both on the sensor node's LCD screen and through the computer software and data has stored in the computer. The result shows that the system is stable and works without any error.

\subsection{Results and Discussions on Power Consumption}

The energy consumption can vary significantly depending on the architecture of the sensor nodes and design technics and components. It is very important that these sensor node components are preferred from components with low energy consumption. Slave (receiver) node's power consumption calculated results show that energy consumption at very low levels in totally for receiver node, even microcontroller energy consumption is negligible. RF receiver unit, RS converter unit and voltage regulator unit spend very low energy and power consumption which is below the acceptable limits. The receiver has two important functions, one of the function is to receive the data from the sensors instantaneously and transfer this data to the computer. Another function is to make in- 
stant surveillance and inspection in the environment and make decisions. The calculated energy consumption with two functions of the designed receiver node are shown in Table 4.

The important finding here, the receiver node will only be used as a passive slave node or will make decisions. If the receiver node is only used to transfer data from the sensor on the network to the computer, the data consumption is very low, the estimated cost is $103.50 \mathrm{~mW}$. If the data from the coming sensors is to be evaluated and transformed into a process, if the PIC microcontroller will decide what to do with the environment check, in this case, the relay unit will be used by the PIC microcontroller on the receiver node, and this will be increased the power consumption too. The estimated cost is $483.50 \mathrm{~mW}$ with relay unit. Another important point is that receiver node will be connected to a computer with cable connection. RS232 to USB converter will be used for transferring sensor data to the computer. Therefore, the receiver node power supply could be used as external supply, for example, $5 \mathrm{~V}$ voltage adapter, battery power is not mandatory for receiver node. So, receiver node power consumption can be negligible. In order to evaluate designed master (transmitter node) in terms of the power consumption, some energy calculations have been made by looking at the technical information of the units such as receiver, voltage regulator, PIC, etc. are used on the designed transmitter nodes.

According to our results, renewable energy sources are needed such as rechargeable battery, supercapacitor and solar panel to use the smoke sensor and gas sensor (MQ2) in wireless sensor networks because of high energy consumption. LM35 Temperature sensor node is usable with non-rechargeable battery due to the least energy consumption. Table 5 shows that obtained energy consumption of the designed transmitter sensor nodes.

The LCD screen is usually not used in wireless sensor networks design to reduce energy consumption and save energy. The sensor network system is monitored via computer software. However, in this sensor node design, the LCD

Table 4. Energy consumption with functions of designed receiver node.

\begin{tabular}{ccc}
\hline \multicolumn{2}{c}{ ENERGY CONSUMPTION OF THE RECEIVER (SLAVE) SENSOR NODE } \\
\hline Modes & With Relay Unit & Without Relay Unit \\
Receiving Mode & $483.50 \mathrm{~mW}$ & $103.50 \mathrm{~mW}$ \\
Working Mode & $452.50 \mathrm{~mW}$ & $72.50 \mathrm{~mW}$ \\
\hline
\end{tabular}

Table 5. Obtained energy consumptions of the designed transmitter nodes.

\begin{tabular}{ccccc}
\hline \multicolumn{4}{c}{ POWER CONSUMPTIONS OF THE TRANSMITTER (MASTER) SENSOR NODES } \\
\hline Modes & DHT11 Node & LDR Node & MQ2 Node & LM35 Node \\
\hline Transmission Mode & $228.50 \mathrm{~mW}$ & $316 \mathrm{~mW}$ & $1016 \mathrm{~mW}$ & $221 \mathrm{~mW}$ \\
Working Mode & $188.50 \mathrm{~mW}$ & $276 \mathrm{~mW}$ & $976 \mathrm{~mW}$ & $181 \mathrm{~mW}$ \\
Idle Mode & $78.50 \mathrm{~mW}$ & $166 \mathrm{~mW}$ & $866 \mathrm{~mW}$ & $71 \mathrm{~mW}$ \\
\hline
\end{tabular}


screen is only used to test the system works and to see the data on the screen. Therefore, Energy consumption of the LCD panel can be ignored.

\subsection{Results and Discussions on the Renewable Energy Integration}

In this study, most of the wireless sensor networks scientific publications and articles are overviewed, only a few studies have considered the integration of renewable energy sources. It is found that in WSNs use of solar panels, rechargeable batteries and supercapacitors are very important in terms of the extending sensor network's lifetime. Table 6 shows that comparison of the previous designed wireless sensor network works in terms of the use of the renewable energy in the last 5 years. One of the major issues in a wireless sensor network is to improve the lifetime of the networks. Against to the increasing energy consumption, contrary to the previous works, serious consideration has been put on renewable energy sources in this study. Designed sensor nodes will be managed energy sources such as rechargeable battery, capacitor and solar energy to survive for long periods of time in WSNs which requires management of the collected environmental energy. Table 6 shows that WSN researchers have gained speed since 2013 and recent works on trends towards to the renewable energy sources is inadequate.

To solve the energy consumption problem in WSN, there is no other way than using renewable energy sources such as solar panel and rechargeable battery. On the use of the renewable energy sources in the WSNs, there are two important constraints. First one is the physical dimensions of the solar panels are still too

Table 6. Comparison of the some previous researches results.

\begin{tabular}{ccccc}
\hline \multicolumn{4}{c}{ Comparison of the Some Previous Researches Since 2013 } & \\
\hline Researchers & Year & $\begin{array}{c}\text { Normal } \\
\text { Battery }\end{array}$ & $\begin{array}{c}\text { Rechargeable } \\
\text { Battery }\end{array}$ & $\begin{array}{c}\text { Super } \\
\text { Capacitor }\end{array}$ \\
\hline Mohanty, Patil [17] & 2013 & $\square$ & X & X \\
Raju, Aravind, Kumar [18] & 2013 & $\square$ & X & X \\
Deshmukh, Pathan, Shaikh [19] & 2013 & $\square$ & X & X \\
Ma,Yan,Wang, Liao, Jin [20] & 2014 & $\square$ & X & X \\
Lambebo, Haghani [21] & 2014 & $\square$ & X & X \\
Nayse, Atique [22] & 2014 & $\square$ & X & X \\
Mohan, Devi [23] & 2014 & $\square$ & X & X \\
Gahlot, Gundkal, Thite [24] & 2015 & $\square$ & X & X \\
Ghayvat, Gui, Suryadevara [25] & 2015 & $\square$ & X & X \\
Tayade, Chandak, Choudhari [26] & 2015 & $\square$ & X & X \\
Pitarma, Marques, Caetano [27] & 2016 & $\square$ & X & X \\
Panic, Stecklina, Stamenkovic [28] & 2016 & $\square$ & X & X \\
Varchola, Drutarovsky [29] & 2017 & $\square$ & X & X \\
Our Platform-Present Work & 2018 & $\square$ & $\square$ & $\square$ \\
\hline
\end{tabular}


large according to the wireless sensor nodes and small size solar cells are not yet able to store enough energy for sensor networks. The second one is the physical dimensions of the rechargeable batteries are still too large which makes node design at micro levels seriously complicated.

\subsection{Results and Discussions Related to Software Issues}

In this study, software has been developed on the Microsoft Visual Studio and C \# language to monitor and save the data in the database with developed software, data could be observed and stored on a computer. Communication between computer observation software and wireless sensor nodes was carried out through RS232 to USB interface. Data collected from the network was stored for later processing purposes. Programmer's software development and PIC coding skills and creativity move the technology in wireless networks forward. The designed receiver node uses the RS232 port for data transfer. This port is a wired communication port. When transmitter sensor nodes are completely wireless (including voltage requirements), the receiver node makes the data transfer wired. So, the cable dependency remains at WSN. Due to the receiver node is wired, it cannot be deployed away from the computer. Therefore, in the feature work, some attention has been given to the wireless transfer of data collected at the receiver node to the computer.

It must be emphasized mention that no research has been conducted on the advantages and disadvantages of wireless data transfer. However, transferring wireless sensor data from the receiving node will enable the sensor networks to operate completely wirelessly and increase the mobility of the system.

\subsection{Other Results and Discussions}

Once the designed system is considered from the aesthetic point of view; the designed wireless sensor node was realized in micro-level. Therefore, the boxing process can be done in small sizes and can give a stylish appearance. One other key feature that should be taken into consideration is weatherproofing. The enclosure is plastic but plastic can be damaged at high temperatures. So, boxes can be built against weather conditions such as temperature, rain, snow, etc. When we consider the designed system in terms of the sustainability; designed sensor nodes are quite stable. However, some design modifications must be made in order to expand on that sustainability and to create a more robust design. For example, solar panel and rechargeable battery must be used to extend sensor node's lifetime, because the designed system supports renewable energy sources as stable.

One other reason why the design is sustainable is the use of add-on wireless sensor nodes. Sensor nodes can be easily swapped and changed out of the environment if needed. Also in time, the price of these sensor nodes component is expected to come down with future releases. When we consider the designed system with mass production; the wireless sensor nodes that can be manufac- 
tured with profits in mind. Because wireless sensor nodes have so many advantages such as ultra-low power energy consumption, lower costs, external voltage modulator renewable energy, using a solar panel and rechargeable battery, micro-level design etc. The sensor node design is considered scientifically system. The miniature design and component properties can be enhanced for mass production.

\section{Conclusions}

This study reviews the utilization of renewable energy to enhance the lifetime of the WSN in environmental applications. So, this study shows the design, testing, and successful development of a renewable energy unit with a supercapacitor, and a rechargeable battery. We have presented a new renewable powered model for the rapid prototyping of WSN. Solar energy clearly outperforms batteries outdoors and can be an alternative for indoor systems. For sensor nodes operating continuously, only outdoor solar power can offer a suitable solution whenever small size is required. The goal of collecting energy from the solar panel by using energy storage components for increasing the efficiency is achieved. Designed WSN was implemented using energy collection, energy storage, energy delivery and energy consumption. Energy schema consists of the solar array which extracts as much as energy to the storages such as batteries and supercapacitor. Designed WSN features the ability to harvest available solar energy from the outdoor environment and deliver this energy to the supercapacitor and battery storage devices. The results of the study would be helpful for the development of WSN with, especially renewable energy sources. WSN has examined in terms of the analytical science and applied science. Our analysis has supported by scientific applications. Studies have conducted on wireless sensor networks based on the realization of low energy and maximum lifetime. The design of the sensor node has supported with ultra-low power nanowatt technology for very low-cost design. Decision-making has created with software algorithm and hardware modules for the implementation of decisions taken by the developed sensor nodes. Developed PIC-based sensor nodes have supported a unique voltage unit with renewable energy sources such as solar panel, rechargeable battery and supercapacitor for energy production and energy-saving. Developed WSN system can be used in industrial applications and daily life applications such as smart factories and smart homes. WSNs can be designed to be used in a wide range. In conclusion, following 4 major goals are achieved in this study:

- Developed a PIC-based wireless sensor node with ultra-low power consumption.

- Developed a PIC-based wireless sensor node with renewable energy sources.

- Developed software for monitoring system and recording data to the database.

- Developed a decision-making in software and hardware for the implementation of decisions taken by the developed sensor nodes. 


\section{References}

[1] Zheng, J. and Jamalipour, A. (2009) Wireless Sensor Networks: A Networking Perspective. IEEE Press, New Jersey, 20-21.

[2] Valada, A., Kohanbash, D. and Kantor, G. (2010) Design and Development of Wireless Sensor Network System for Precision Agriculture. Robotics Institute, Carnegie Mellon University, Pittsburgh, Pennsylvania, 19.

[3] Voigt, T., Ritter, H. and Schiller, J. (2004) Utilizing Solar Power in Wireless Sensor Networks. Freie Universität Berlin, Germany, Institut für Informatik, 1-9.

[4] Martino, M. and Varley, J. (2012) A Wireless Sensor Node Powered by a PV/Supercapacitor/Battery Trio, The Edward S. Rogers Sr., Department of Electrical and Computer Engineering, University of Toronto, ECE496Y Design Project Course 3-89.

[5] Soylu, T. (2011) Wireless Sensor Networks Applications and Design of Sensor Node. Master Thesis, Trakya University, Institute of Science, Edirne, 144.

[6] Ozcan, O. (2011) PIC Based Sensor for Wireless Sensor Network Node Design. Master Thesis, Selcuk University, Institute of Science, Konya, 29.

[7] Shaker, M. and Imran, A. (2013) Greenhouse Micro Climate Monitoring Based on WSN with Smart Irrigation Technique. World Academy of Science, Engineering and Technology International Journal of Electrical, Computer, Energetic, Electronic and Communication Engineering, 7.

[8] MICROCHIP Technology Inc. (2007) PIC16F882/883/884/886/887 Data Sheet, 28/40/44-Pin, Enhanced Flash-Based 8-Bit CMOS Microcontrollers with Nanowatt, 4.

[9] SHENZHEN EONE Electronics Company Ltd. (2009) 1602A-1 LCD Module Specification Ver1.0, 2.

[10] Johnson, M., Healy, M., Ven, P., Hayes, M.J., Nelson, J., Newe T. and Lewis, E. (2009) A Comparative Review of Wireless Sensor Network Mote Technologies. 2009 IEEE SENSORS, 25-28 October 2009, Christchurch, New Zealand, 1440. https://doi.org/10.1109/ICSENS.2009.5398442

[11] Beutel, J. (2006) Metrics for Sensor Network Platforms. Proceedings of ACM Workshop on Real-World Wireless Sensor Networks (REALWSN06), New York, 26-30.

[12] DHT11 Humidity \& Temperature Sensor (2010) DHT11 Temperature \& Humidity Sensor Features a Temperature \& Humidity Sensor Complex with a Calibrated Digital Signal Output, D-Robotics UK, 1-3. http://www.droboticsonline.com

[13] Texas Instruments (2016) LM35 Precision Centigrade Temperature Sensors, SNIS159G-AUGUST 1999-REVISED, 1.

[14] HANWEI Electronics, Co., LTD. (2017) Technical Data MQ-2 Gas Sensor, 1. http://www.hwsensor.com/

[15] Semiconductor Components Industries LLC (2016) Literature Distribution Center for ON Semiconductor, JRev. 14, Publication Order Number: LM317/D, Colorado, USA. http://www.onsemi.com

[16] Gray, C. and Campbell, L. (2016) Towards an Energy-Efficient Internet of Things. 26th International Telecommunication Networks and Applications Conference, 29.

[17] Mohanty, N.R. and Patil, C.Y. (2013) Wireless Sensor Networks Design for Greenhouse Automation. International Journal of Engineering and Innovative Technology, 3, 257-262. 
[18] Raju, P.V., Aravind, R.V.R.S. and Kumar, B.S. (2013) Pollution Monitoring System using Wireless Sensor Network in Visakhapatnam. International Journal of Engineering Trends and Technology, 4, 591-595. http://www.ijettjournal.org

[19] Mane-Deshmukh, P.V., Ladgaonkar, B.P., Pathan, S.C. and Shaikh, S.S. (2013) Microcontroller Pic $18 \mathrm{f} 4550$ Based Wireless Sensor Node to Monitor Industrial Environmental Parameters. International Journal of Advanced Research in Computer Science and Software Engineering, 3, 943-950.

[20] Ma, L., Yan, J., Yan, S., Wang, B., Liao, K., Jin and Wang, J. (2014) A Study of Agricultural Meteorological Monitoring System Based on Wireless Sensor Network. International Journal of Multimedia and Ubiquitous Engineering, 9, 15-26. https://doi.org/10.14257/ijmue

[21] Lambebo, A. and Haghani, S. (2014) A Wireless Sensor Network for Environmental Monitoring of Greenhouse Gases. University of Bridgeport, Bridgeport, 1-4.

[22] Nayse, S.P. and Atique, M. (2014) Design of Application Based Wireless Sensor Node. 2014 Federated Conference on Computer Science and Information Systems, Vol. 3, Warsaw, 7-10 September 2014, 177-181. https://doi.org/10.15439/2014F508

[23] Mohan, W.S. and Devi, R.J. (2014) Monitoring of Geological $\mathrm{CO}_{2}$, Based on Wireless Sensor Networks. International Journal of Engineering Sciences \& Research Technology, 595-600. http://www.ijesrt.com/

[24] Gahlot, N., Gundkal, V., Kothimbire, S. and Thite, A. (2015) Zigbee Based Weather Monitoring System. The International Journal of Engineering and Science, 4, 61-66.

[25] Ghayvat, H., Mukhopadhyay, S., Gui, X. and Suryadevara, N. (2015) WSN- and IOT-Based Smart Homes and Their Extension to Smart Buildings. Sensors (Basel), 15, 10350-10379. http://www.mdpi.com/journal/sensors https://doi.org/10.3390/s150510350

[26] Tayade, S.B., Chandak, D.S. and Choudhari, P.S. (2015) Real-Time Monitoring of Geological $\mathrm{CO}_{2}$ Storage and Leakage Using Wireless Sensor Network. Geological $\mathrm{CO}_{2}$ Leakage Monitoring Equipment Based on Wireless Sensors. Journal of Innovative and Emerging Research in Engineering, 2, 15-21.

[27] Pitarma, R., Marques, G. and Caetano, F. (2016) Monitoring Indoor Air Quality to Improve Occupational Health. In: Rocha, Á., et al., Eds., New Advances in Information Systems and Technologies, Advances in Intelligent Systems and Computing 445, Springer International Publishing, Berlin, 13-21.

[28] Panic, G., Stecklina, O. and Stamenkovic, Z. (2016) An Embedded Sensor Node Microcontroller with Crypto-Processors. Sensors, 16, E607.

http://www.mdpi.com/journal/sensors https://doi.org/10.3390/s16050607

[29] Varchola, M. and Drutarovsky, M. (2007) Zigbee Based Home Automation Wireless Sensor Network. Acta Electrotechnica et Informatica, 7, 1-8. 